\begin{tabular}{lll}
\hline Utijuuihluw & 65, №3, 2012 & Механика
\end{tabular}

\title{
ABOUT THE STRESS STATE OF A PIECEWISE HOMOGENEOUS ELASTIC RECTANGLE WITH TWO SYMMETRIC CENTRAL CRACKS IN ANTIPLANE DEFORMATIONS Pahlaviani Ali G.
}

Keywords: Piecewise homogeneous Cracked Plate, bimaterial interface Cracking, Antiplane S.I.F, Tearing mode.

Ключевые слова: кусочно-однородный прямоугольник, антиплоская деформация, разрывная мода.

Пахлавиани Али Г.

О напряжённом состоянии кусочно-однородного упругого прямоугольника с двумя симметричными трещинами при антиплоской деформации

В статье в явном виде определяются коэффициенты интенсивности напряжений, разрушающие касательные напряжения и раскрытия трещин в кусочно-однородном прямоугольнике с двумя симметричными трещинами при антиплоской деформации. Численным анализом выяснены закономерности изменения этих величин, а также эффекты взаимовлияний ближних концов трещин.

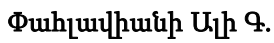

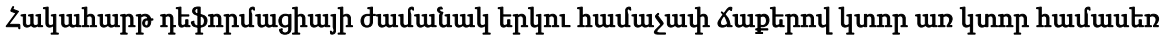

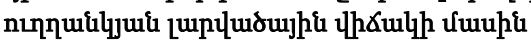

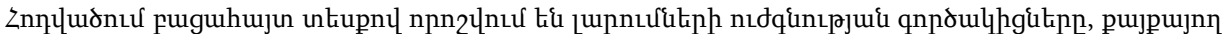

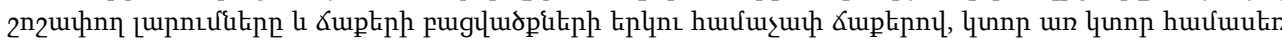

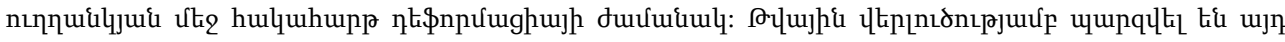

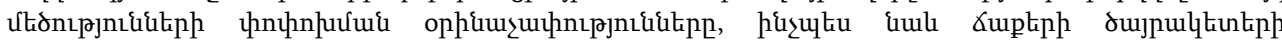
hnjuuqnignıpjui thtilunitinn:

\section{Introduction}

This paper presents a boundary value problem and formulation for the analysis of linear elastic fracture mechanics problems involving piecewise homogeneous bimaterials.

Investigation and mathematical works in this paper is focused on the stress- strain state of plates with two symmetric central cracks that are made of two bonded dissimilar materials which behave as a piecewise homogeneous elastic plate. Two cracks are on the bondness line of the two segments of plate that have two different shear modulus G1 and G2, and equal length and height. The antiplane distributed shear loading act on the edges of plate. Using sinusoidal Fourier transformation the equation governing this boundary value problem converts to a singular integral equation (S.I.E) of the Cauchy form, which can be solved with the aid of Gaussian numerical-analytical solution for singular integral equations. Consequently the dislocation field around the cracks boundaries and the tearing stresses of plate and the Stress Intensity Factor (S.I.F) equations at the tips of cracks are derived.

\section{Basic equations in bimaterials with two cracks}

We consider the rectangular plate in the Cartesian coordinate system $O x y z$, has a upper part $D_{1}=\{0 \leq x \leq l, 0 \leq y \leq h\}$ with shear modulus $G_{1}$, length $l$ and height $h$, and a 
lower part $D_{2}=\{0 \leq x \leq l,-h \leq y \leq 0\}$ with shear modulus $G_{2}$, length $l$ and height $h$. On the bondness line of the two segments in the interval $0 \leq x \leq l$ there are two central cracks located symmetrically at $L=\bigcup_{k=1}^{2}\left[a_{k}, b_{k}\right]$, that have equal lengths (Fig.1) $a_{1}=\frac{l}{2}-a, b_{1}=\frac{l}{2}-b, a_{2}=\frac{l}{2}+b, b_{2}=\frac{l}{2}+a, \quad(0<a<l / 2 ; \quad b<a)$

The boundaries of cracks have not tractions, furthermore the vertical edges of plate $D_{j}(j=1,2)$ at $x=0$ and $x=l$ are clamped and the upper and lower horizontal edges $y= \pm h$ are loaded by antiplane distributed shear traction $T(x)$, so that we have $\left.\tau_{y z}^{(1)}\right|_{y=h-0}=\left.\tau_{y z}^{(2)}\right|_{y=-h+0}=T(x)(0<x<1)$, in which $\tau_{y z}^{(j)}(j=1,2)$ are the antiplane shear stresses on the top and bottom boundaries of segments $D_{j}$. According to the above assumptions, the rectangular plate $D=\bigcup_{k=1}^{2} D_{k}$ undergoes an antiplane strain situation and the deformations of crack edges occur along the $O z$ axis on the basis of plane $O x y$ which means a tearing mode of Fracture. In this problem we seek to determine the displacement of edges around the cracks boundaries $L$, the stress intensity factors S.I.F, and the plate shear stresses producing fracture at the bondness line $L^{\prime}=[0, l] \backslash L$ out of cracks. Moreover it is required to investigate the interactive influence of adjacent crack tips $a_{2}$ and $b_{1}$.

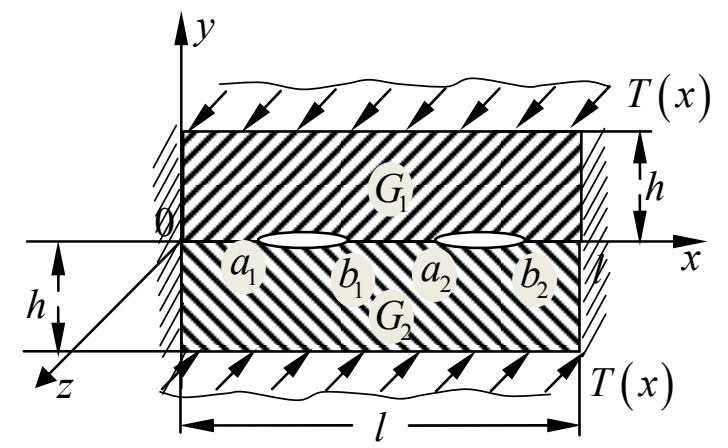

Fig.1

For determination of governing equations of the problem as shown in [10], in which the general form of the problem was discussed, the piecewise homogeneous elastic plate $D$ in interval $0 \leq x \leq l$ of axis $O x$ is investigated separately in upper rectangle $\left(D_{1}\right)$ and lower rectangle $\left(D_{2}\right)$. By means of function $w_{j}(x, y) \quad(j=1,2)$ we introduce the deformation of cracks boundaries of $D_{j}(j=1,2)$ along the $O z$ direction and investigate their elastic equilibrium separately. So for rectangle $D_{1}$ using the Hooke's law to calculate the displacement $w_{1}(x, y)$ we reach to the boundary value problem $(1.1)$ : 


$$
\begin{aligned}
& \Delta w_{1}=\frac{\partial^{2} w_{1}}{\partial x^{2}}+\frac{\partial^{2} w_{1}}{\partial y^{2}}=0 ; \quad(0<x<l, 0<y<h) \\
& \left.w_{1}(x, y)\right|_{x=0}=\left.w_{1}(x, y)\right|_{x=l}=0 ; \quad(0<y<h) \\
& \left\{\left.\tau_{y z}^{(1)}\right|_{y=+0}=\left.G_{1} \frac{\partial w_{1}}{\partial y}\right|_{y=+0}=\tau_{+}(x) ;\left.\quad \tau_{y z}^{(1)}\right|_{y=h-0}=\left.G_{1} \frac{\partial w_{1}}{\partial y}\right|_{y=h-0}=T(x) ; \quad(0<x<l)\right. \\
& \left.\tau_{y z}^{(1)}\right|_{y=+0}=\tau_{+}(x)= \begin{cases}0 & \left(x \in L^{+}\right) ; \\
\tau(x) & \left(x \in L^{\prime}\right) ;\end{cases}
\end{aligned}
$$

$L^{+}$is upper edge of the cracks $L$, and $\tau(x)$ is the antiplane shear stress in non-cracked zone of the plate $L^{\prime}=[0, l] \backslash L$.

After [11] to solve the boundary value problem (1.1) the Fourier sinusoidal transformation is used, supposing that

$\bar{w}_{1}(n, y)=\int_{0}^{l} w_{1}(x, y) \sin \left(\frac{\pi n x}{l}\right) d x \quad(n=1,2 \ldots)$

And Fourier inverse transformation as below

$w_{1}(x, y)=\frac{2}{l} \sum_{n=1}^{\infty} \bar{w}_{1}(n, y) \sin \left(\frac{\pi n x}{l}\right) \quad(0 \leq x \leq l)$.

By means of Fourier transformation (1.3) in the boundary value problem (1.1), the ordinary differential equation is obtained:

$$
\begin{aligned}
& \left\{\frac{d^{2} \bar{w}_{1}}{d y^{2}}-\frac{\pi^{2} n^{2}}{l^{2}} \bar{w}_{1}=0 \quad(0<y<h)\right. \\
& \left\{\left.G_{1} \frac{d \bar{w}_{1}}{d y}\right|_{y=+0}=\bar{\tau}_{+}(n),\left.\quad G_{1} \frac{d \bar{w}_{1}}{d y}\right|_{y=h}=\bar{T}(n)\right. \\
& \bar{\tau}_{+}(n)=\int_{0}^{l} \tau_{+}(x) \sin \left(\frac{\pi n x}{l}\right) d x, \quad \bar{T}(n)=\int_{0}^{l} T(x) \sin \left(\frac{\pi n x}{l}\right) d x \quad(n=1,2 \ldots)
\end{aligned}
$$

$$
\begin{aligned}
& \text { Solving the equation (1.4) leads to } \quad(n=1,2, \ldots) \quad(0 \leq y \leq h) \\
& \bar{w}_{n}(n, y)=\frac{l}{\pi n G_{1} \sin (\pi n h / l)}\left\{\bar{T}(n) \operatorname{ch}\left(\frac{\pi n y}{l}\right)-\bar{\tau}_{+}(n) \operatorname{ch}[\pi n(y-h) / l]\right\}
\end{aligned}
$$

Finally from which we obtain the deformations on the bondness line upper boundaries

$$
\bar{w}_{1}(n, 0)=\frac{l\left[\bar{T}(n)-\bar{\tau}_{+}(n) \operatorname{ch}(\pi n h / l)\right]}{\pi n G_{1} \operatorname{sh}(\pi n h / l)} \quad(n=1,2, \ldots) .
$$

Completely through a similar approach for the rectangle $D_{2}$, we obtain the deformation on the bondness line

$$
\bar{w}_{2}(n, 0)=\frac{l\left[\bar{\tau}_{-}(n) \operatorname{ch}(\pi n h / l-\bar{T}(n))\right]}{\pi n G_{2} \operatorname{sh}(\pi n h / l)} \quad(n=1,2, \ldots),
$$

it is clear that 


$$
\begin{aligned}
& \left\{\bar{w}_{2}(n, y) ; \bar{\tau}_{-}(n)\right\}=\int_{0}^{l}\left\{w_{2}(x, y) ; \tau_{-}(x)\right\} \sin \left(\frac{\pi n x}{l}\right) d x \\
& \tau_{-}(x)=\tau_{+}(x)=\left.\tau_{y z}^{(1)}\right|_{y=+\Delta}=\left.\tau_{y z}^{(2)}\right|_{y=-0}= \begin{cases}0 & \left(x \in L^{-}\right) \\
\tau(x) & \left(x \in L^{\prime}\right) .\end{cases}
\end{aligned}
$$

In which the zone $L^{-}$is the lower boundaries of cracks $L$.

Now using $w_{1}(x, 0), w_{2}(x, 0)$ quantities, we introduce the following functions:

$$
\begin{aligned}
& \Phi(x)=\frac{w_{1}(x, 0)-w_{2}(x, 0)}{2}=w_{1}(x, 0)=\frac{2}{l} \sum_{n=1}^{\infty} \Phi_{n} \sin \left(\frac{\pi n x}{l}\right) \quad(0<x<l) \\
& \Omega(x)=\frac{\tau_{+}(x)+\tau_{-}(x)}{2}=\tau_{+}(x)=\frac{2}{l} \sum_{n=1}^{\infty} \Omega_{n} \sin \left(\frac{\pi n x}{l}\right)
\end{aligned}
$$

Functions $\Phi_{n}, \Omega_{n}$ are the Fourier Sinus coefficients that according to relations (1.2), (1.3) and (1.5) presented as below:

$$
\Phi_{n}=\frac{1}{2}\left[\bar{w}_{1}(n, 0)-\bar{w}_{2}(n, 0)\right]=\bar{w}_{1}(n, 0) ; \quad \Omega_{n}=\frac{1}{2}\left[\bar{\tau}_{+}(n)+\bar{\tau}_{-}(n)\right]=\bar{\tau}_{+}(n) .
$$

In those it is clear that due to symmetry of axis $O x$

$$
w_{1}(x, y)=-w_{2}(x, y) \quad(0 \leq x \leq 1 ;-h \leq y \leq h), \tau_{+}(x)=\tau_{-}(x) \text {. }
$$

Now regarding to (1.6)-(1.9) and using similar approach in [10] with using nondimensional parameters $\eta, \xi$ we convert the function $\Omega(x)$ from (1.8) to the following formula.

$$
\begin{array}{ll}
\Omega_{0}(\xi)=-\frac{1}{2 \pi} \int_{L_{0}}\left(\operatorname{ctg} \frac{\xi-\eta}{2}+\operatorname{ctg} \frac{\xi+\eta}{2}\right) \phi_{0}(\eta) d \eta+ & \\
+\frac{1}{\pi} \int_{L_{0}}\left[K_{*}(\xi-\eta)+K_{*}(\xi+\eta)\right] \phi_{0}(\eta) d \eta+ & (0<\xi<\pi) \\
+\frac{2}{\pi} \int_{0}^{\pi}\left[L_{*}(|\xi-\eta|)-L_{*}(\xi+\eta)\right] T_{0}(\eta) d \eta &
\end{array}
$$

So that the first term in the first integral for $\eta=\xi$ is the main quantity of Cauchy function, for this reason we use the below functions in (1.10).

$$
K_{*}(\xi)=\sum_{n=1}^{\infty} \frac{e^{-n h_{0}} \sin (n \xi)}{\operatorname{ch}\left(n h_{0}\right)} ; \quad L_{*}(\xi)=\sum_{n=1}^{\infty} \frac{\cos (n \xi)}{\operatorname{ch}\left(n h_{0}\right)} \quad(0<\xi<\pi)
$$

Introducing nondimensional parameters converts the interval $L$ to $L_{0}$, so with the aid of function $\Phi(x)$ from (1.8), the cracks boundaries displacement field are derived as below:

$$
\begin{aligned}
& \xi=\frac{\pi x}{l}, \eta=\frac{\pi s}{l}, h_{0}=\frac{\pi h}{l} ; \bar{\tau}(\xi)=G_{*} \tau(l \xi / \pi), T_{0}(\xi)=G_{*} T(l \xi / \pi) \\
& G_{*}=\frac{G_{1}+G_{2}}{2 G_{1} G_{2}}, \quad \alpha_{k}=\frac{\pi a_{k}}{l}, \beta_{k}=\frac{\pi b_{k}}{l}(k=1,2),
\end{aligned}
$$




$$
\begin{aligned}
& L_{0}=\bigcup_{k=1}^{2}\left[\alpha_{k}, \beta_{k}\right], \quad L_{0}^{\prime}=[0, \pi] \backslash L_{0}, \quad 0<\xi, \quad \eta<\pi . \\
& \Phi^{\prime}(x)=\left\{\begin{array}{ll}
\varphi^{\prime}(x) & (x \in L) ; \\
0 & \left(x \in L^{\prime}\right) ;
\end{array}=\frac{2}{l} \sum_{n=1}^{\infty} \varphi_{n} \cos (n \xi),\right. \\
& \varphi_{n}=\frac{\pi n}{l} \Phi_{n}=\frac{l}{\pi} \int_{L_{0}} \varphi_{0}(\xi) \cos (n \xi) d \xi ; \varphi_{0}(\xi)=\varphi^{\prime}(l \xi / \pi) .
\end{aligned}
$$

The cracks $L$ geometry parameters are $\left(0<\alpha<\frac{\pi}{2}, \beta<\alpha\right)$.

$\alpha_{1}=\frac{\pi}{2}-\alpha, \beta_{1}=\frac{\pi}{2}-\beta, \alpha_{2}=\frac{\pi}{2}+\beta, \beta_{2}=\frac{\pi}{2}+\alpha, \alpha=\frac{\pi a}{l}, \quad \beta=\frac{\pi b}{l}$.

Investigation of key equation (1.10) in interval $L_{0}$ leads us to below new variables $t=\cos \xi, u=\cos \eta \quad(-1<t, u<1)$,

Regarding to the unknown displacement field $\varphi_{0}(\xi)$ from equation (1.12), we can derive the singular integral equation governing the problem as follows

$$
\begin{gathered}
\frac{1}{\pi} \int_{\Lambda_{0}} \frac{\omega_{0}(u) d u}{u-t}+\frac{1}{\pi} \int_{\Lambda_{0}}\left[\frac{(u+t) K_{0}(t, u)}{\sqrt{1-u^{2}}}-2 K(t, u)\right] \omega_{0}(u) d u=f(t)\left(t \in \Lambda_{0}\right) \\
f(t)=\frac{2}{\pi} \int_{-1}^{1} L(t, u) \bar{T}_{0}(u) d u ; \quad \bar{T}_{0}(t)=T_{0}(\arccos t) ; \quad \Lambda_{0}=\bigcup_{k=1}^{2}\left[\delta_{k}, \gamma_{k}\right] ; \\
\omega_{0}(t)=\varphi_{0}(\arccos t) ; \quad K_{0}(t, u)=\left(\sqrt{1-t^{2}}+\sqrt{1-u^{2}}\right)^{-1}
\end{gathered}
$$

And according to (1.13) we can write:

$\delta_{1} \cos \beta_{2}=-\sin \alpha ; \gamma_{1}=\cos \alpha_{2}=-\sin \beta$;

$\delta_{2}=\cos \beta_{1}=\sin \beta ; \quad \gamma_{2}=\cos \alpha_{1}=\sin \alpha$.

Moreover, based on relations (1.11), we use the below functions:

$$
\begin{aligned}
& K(t, u)=\frac{\sqrt{1-t^{2}}}{\sqrt{1-u^{2}}} \sum_{n=1}^{\infty} \frac{e^{-n h_{0}}}{\operatorname{ch}\left(n h_{0}\right)} T_{n}(u) U_{n-1}(t) \quad(-1<t, u<1) \\
& L(t, u)=\sqrt{1-t^{2}} \sum_{n=1}^{\infty} \frac{U_{n-1}(u) U_{n-1}(t)}{\operatorname{ch}\left(n h_{0}\right)} ;
\end{aligned}
$$

That are the known Chebyshev polynomials of the first and the second kind.

The singular integral equation (1.14)-(1.16) may be investigated at the tips of the cracks to find their mutual influences. The continuity condition is:

$\int_{a_{k}}^{b_{k}} \varphi^{\prime}(s) d s=0 \quad(k=\overline{1,2})$

Using the nondimensional parameters, the above condition relation differ to below form:

$\int_{\delta_{k}}^{\gamma_{k}} \frac{\omega_{0}(u) d u}{\sqrt{1-u^{2}}}=0 \quad(k=\overline{1,2})$ 
Through solution of the singular integral equation (1.14)-(1.16) with the condition (1.17), and after investigation the key equation (1.10) in the zone out of the cracks, $L_{0}^{\prime}=[0, \pi] \backslash L_{0}$, we derive the nondimensional relation for shear stresses $\bar{\tau}(\xi) \quad(\xi=\arccos t)$ as follows

$\tau_{0}(t)=\bar{\tau}(\arccos t)=-\frac{1}{\pi} \int_{\Lambda_{0}} \frac{\omega_{0}(u) d u}{u-t}-\frac{1}{\pi} \int_{\Lambda_{0}}\left[\frac{u+t}{\sqrt{1-u^{2}}} K_{0}(t, u)-\right.$

$-2 K(t, u)] \omega_{0}(u) d u+\frac{2}{\pi} \int_{-1}^{1} L(t, u) \bar{T}_{0}(u) d u \quad\left(t \in[-1,1] \backslash \Lambda_{0}\right)$

In which the functions $K_{0}(t, u), K(t, u), L(t, u)$ and $\bar{T}_{0}(t)$ are defined according to formulas (1.14) and (1.16).

\section{Reducing the S.I.E to a system of linear algebraic equations}

To solve the S.I.E (1.14)-(1.17) we use the numerical solutions and methods prescribed in [7-9] that are based on Gaussian Quadratic formulas for ordinary and singular integrals. To reach this main object, at the end points of each interval $\left[\delta_{k}, \gamma_{k}\right](k=1,2)$ of crack systems $\Lambda_{0}$, we perform a change of variables according the below formulas

$t=\frac{\gamma_{k}-\delta_{k}}{2} r+\frac{\gamma_{k}+\delta_{k}}{2} ; u=\frac{\gamma_{k}-\delta_{k}}{2} \rho+\frac{\gamma_{k}+\delta_{k}}{2} \quad(k=1,2)$

which is valid in interval $[-1,1]$ where $-1 \leq r, \rho \leq 1$. From that and taking advantage relations $(1.15)$ it is obtained

$t=\frac{\sin \alpha-\sin \beta}{2} r-\frac{\sin \alpha+\sin \beta}{2} ; u=\frac{\sin \alpha-\sin \beta}{2} \rho-\frac{\sin \alpha+\sin \beta}{2}(k=1)$

$t=\frac{\sin \alpha-\sin \beta}{2} r+\frac{\sin \alpha+\sin \beta}{2} ; u=\frac{\sin \alpha-\sin \beta}{2} \rho+\frac{\sin \alpha+\sin \beta}{2}(k=2)$

Consequently the S.I.E (1.14)-(1.16) is converted to the form as below

$$
\begin{aligned}
& \frac{1}{\pi} \int_{-1}^{1} \frac{\omega_{k}^{(0)}(\rho) d \rho}{\rho-r}+\frac{1}{\pi} \int_{-1}^{1} L_{k n}(r, \rho) \omega_{n}^{(0)} d \rho+\frac{1}{2 \pi} \sum_{n=1}^{2} \int_{-1}^{1} K_{k n}(r, \rho) \omega_{n}^{(0)}(\rho) d \rho=f_{k}(r) ; \\
& f_{k}(r)=f\left(\frac{\gamma_{k}-\delta_{k}}{2} r+\frac{\gamma_{k}+\delta_{k}}{2}\right) ;(k=1,2-1<r<1) \\
& L_{k n}(r, \rho)=\left(\rho-\frac{\gamma_{k}-\delta_{k}}{\gamma_{n}-\delta_{n}} r+\frac{\gamma_{n}+\delta_{n}}{\gamma_{n}-\delta_{n}}-\frac{\gamma_{k}+\delta_{k}}{\gamma_{n}-\delta_{n}}\right)^{-1} ; \\
& K_{k n}(r, \rho)=\left(\gamma_{n}-\delta n\right)\left\{\frac{\left(\gamma_{n}-\delta_{n}\right) \rho+\left(\gamma_{k}-\delta_{k}\right) r+\gamma_{n}+\delta_{n}+\gamma_{k}+\delta_{k}}{\sqrt{4-\left[\left(\gamma_{n}-\delta_{n}\right) \rho+\gamma_{n}+\delta_{n}\right]^{2}} \times}\right. \\
& \times K_{0}\left(\frac{\gamma_{k}-\delta_{k}}{2} r+\frac{\gamma_{k}+\delta_{k}}{2}, \frac{\gamma_{n}-\delta_{n}}{2} \rho+\frac{\gamma_{n}+\delta_{n}}{2}\right)-2 K\left(\frac{\gamma_{k}-\delta_{k}}{2} r+2\right) \\
& \left.\left.+\frac{\gamma_{k}+\delta_{k}}{2}, \frac{\gamma_{n}-\delta_{n}}{2} \rho+\frac{\gamma_{n}+\delta_{n}}{2}\right)\right\} ; \omega_{k}^{(0)}(r)=\omega_{0}\left(\frac{\gamma_{k}-\delta_{k}}{2} r+\frac{\gamma_{k}+\delta_{k}}{2}\right), \quad(k=1,2)
\end{aligned}
$$


where the function $f(t)$ and kernel function $K_{0}(t, u)$ and $K(t, u)$ are introduced according to formulas (1.14) and (1.16), and the end points of crack systems $\Lambda_{0}=\bigcup_{k=1}^{2}\left[\delta_{k}, \gamma_{k}\right]$ defined by (1.15) quantities. On the other hand noting that $k=1$ and $k=2$ the relations (2.1) are valid, and making use of (2.1) the condition equation (1.17) is converted as below:

$\int_{-1}^{1} \frac{\omega_{k}^{(0)}(\rho) d \rho}{\sqrt{4-\left[\left(\gamma_{k}-\delta_{k}\right) \rho+\left(\gamma_{k}+\delta_{k}\right)\right]^{2}}}=0 \quad(k=1,2)$.

Now through the numerical approach according to [10] we can solve the S.I.E (2.2) considering the above continuity condition (2.3) to obtain a system of linear algebraic equations as follows :

$$
\begin{aligned}
& \left\{\begin{array}{l}
\sum_{p=1}^{M} \frac{1}{M}\left[\frac{1}{\rho_{p}-r_{m}}+L_{k n}\left(r_{m}, \rho_{p}\right)+\frac{1}{2} \sum_{n=1}^{2} K_{k n}\left(r_{m}, \rho_{p}\right)\right] \psi_{n}^{(0)}\left(\rho_{p}\right)=\underset{(m=1, M-1, k=1,2)}{f_{k}\left(r_{m}\right)} \\
\sum_{p=1}^{M} \frac{\psi_{k}^{(0)}\left(\rho_{p}\right)}{M \sqrt{1-\left(\frac{\gamma_{k}-\delta_{k}}{2} \rho_{p}+\frac{\gamma_{k}+\delta_{k}}{2}\right)^{2}}}=0
\end{array}\right. \\
& \omega_{k}^{(0)}=\frac{\psi_{k}^{(0)}(\rho)}{\sqrt{1-\rho^{2}}}(k=1,2),
\end{aligned}
$$

For this reason in equations (2.2) and (2.3) the below function is valid where $\psi_{k}(\rho)$ is a function of Holder-Lipschitz class in the $[-1,1]$ range, and $M$ is an arbitrary natural numbers, also $r_{m}$ and $\rho_{p}$ are the roots of Chebyshev polynomials of the second kind $U_{m-1}(r)$ and the first kind $T_{m}(\rho)$.

$r_{m}=\cos \left(\frac{\pi m}{M}\right)(m=\overline{1, M-1}) ; \rho_{p}=\cos \left(\frac{2 p-1}{2 M} \pi\right)(p=\overline{1, M})$

\section{Special case of antiplane loading}

Now we suppose that the upper and lower edges of the plate stressed by antiplane shear forces $\mathrm{P}$ so that $T(x)=P \delta(x-l / 2)$, in which $\delta(x)$ is the known Dirac Delta function.

By this assumption the function $f(t)$ is calculated as below:

$$
\begin{aligned}
& f(t)=Q_{0} \sqrt{1-t^{2}} \sum_{n=1}^{\infty} \frac{(-1)^{n-1}}{\operatorname{ch}\left[(2 n-1) h_{0}\right]} U_{2 n-2}(t) \quad(-1<t<1) \\
& Q_{0}=P\left(G_{1}+G_{2}\right) / G_{1} G_{2} l .
\end{aligned}
$$

To calculate the shear stress from relations (1.18) making use of the variables shown in (2.1) we reach to a new variable $\rho$ and finally taking into consideration the equation (2.5) the follow formula for shear stress is concluded 


$$
\begin{aligned}
& \tau_{0}(t)=-\frac{1}{2 \pi} \sum_{k=1}^{2}\left(\gamma_{k}-\delta_{k}\right) \int_{-1}^{1} \frac{\psi_{k}^{(0)}(\rho) d \rho}{\sqrt{1-\rho^{2}}\left(\frac{\gamma_{k}-\delta_{k}}{2} \rho+\frac{\gamma_{k}+\delta_{k}}{2}-t\right)}- \\
& -\frac{1}{2 \pi} \sum_{k=1}^{2}\left(\gamma_{k}-\delta_{k}\right) \int_{-1}^{1}\left[\frac{\left(\gamma_{k}-\delta_{k}\right) \rho+\gamma_{k}+\delta_{k}+2 t}{\sqrt{4-\left[\left(\gamma_{k}-\delta_{k}\right) \rho+\gamma_{k}+\delta_{k}\right]^{2}}} \times\right. \\
& \times K_{0}\left(t, \frac{\gamma_{k}-\delta_{k}}{2} \rho+\frac{\gamma_{k}+\delta_{k}}{2}\right)-2 K\left(t, \frac{\gamma_{k}-\delta_{k}}{2} \rho+\frac{\gamma_{k}+\delta_{k}}{2}\right) \frac{\psi_{k}^{(0)}(\rho) d \rho}{\sqrt{1-\rho^{2}}}+f(t) \\
& \quad\left(t \in[-1,1] \backslash \Lambda_{0}\right) ; \Lambda_{0}=[-\sin \alpha,-\sin \beta] \bigcup[\sin \beta, \sin \alpha]
\end{aligned}
$$

in which the function $f(t)$ is presented according to (3.1). Consequently we can obtain the nondimensional fracture stresses through solving the linear algebraic system (2.4) that is:

$$
\begin{aligned}
& \tau_{0}(t)=-\frac{1}{2 M} \sum_{k=1}^{2}\left(\gamma_{k}-\delta_{k}\right) \sum_{p=1}^{M} \frac{\psi_{k}^{(0)}\left(\rho_{p}\right)}{\frac{\gamma_{k}-\delta_{k}}{2} \rho_{p}+\frac{\gamma_{k}+\delta_{k}}{2}-t}- \\
& -\frac{1}{2 M} \sum_{k=1}^{2}\left(\gamma_{k}-\delta_{k}\right) \sum_{p=1}^{M} \frac{\left(\gamma_{k}-\delta_{k}\right) \rho_{p}+\gamma_{k}+\delta_{k}+2 t}{\sqrt{4-\left[\left(\gamma_{k}-\delta_{k}\right) \rho_{p}+\gamma_{k}+\delta_{k}\right]^{2}}} \times \\
& \times K_{0}\left(t, \frac{\gamma_{k}-\delta_{k}}{2} \rho_{p}+\frac{\gamma_{k}+\delta_{k}}{2}\right)-2 K\left(t, \frac{\gamma_{k}-\delta_{k}}{2} \rho_{p}+\frac{\gamma_{k}+\delta_{k}}{2}\right) \psi_{k}^{(0)}\left(\rho_{p}\right)+ \\
& +f(t) \quad\left(t \in[-1,1] \backslash \Lambda_{0}\right)
\end{aligned}
$$

Now we seek for a relation to represent the crack opening displacements $\Psi(x)$ :

$$
\Psi(x)=2 \Phi(x)=2 \int_{a_{k}}^{x} \varphi^{\prime}(s) d s=-2 \int_{x}^{b_{k}} \varphi^{\prime}(s) d s . \quad\left(a_{k} \leq x \leq b_{k} ; k=1,2\right)
$$

From which after some calculations we have $(-1 \leq r \leq 1 ; k=1,2)$

$$
\begin{aligned}
& \Psi_{k}^{(0)}(r)=-\frac{\gamma_{3-k}-\delta_{3-k}}{2 \pi} \int_{-1}^{1} \frac{\operatorname{sign}(r-\rho) \Psi_{3-k}^{(0)}(\rho) d \rho}{\sqrt{1-\rho^{2}} \sqrt{1-\left(\frac{\gamma_{3-k}-\delta_{3-k}}{2} \rho+\frac{\gamma_{3-k}+\delta_{3-k}}{2}\right)^{2}}} \\
& \Psi_{k}^{(0)}(r)=\frac{1}{l} \Psi\left(\frac{1}{\pi} \arccos \frac{\gamma_{3-k}-\delta_{3-k}}{2} r+\frac{\gamma_{3-k}+\delta_{3-k}}{2}\right)
\end{aligned}
$$

Similar through solving the system (2.4), the nondimensional equation (3.4) for crack opening displacements is obtained as below $(-1 \leq r \leq 1 ; k=1,2)$ : 


$$
\Psi_{k}^{(0)}(r)=-\frac{\gamma_{3-k}-\delta_{3-k}}{2 M} \sum_{p=1}^{M} \frac{\operatorname{sign}\left(r-\rho_{p}\right) \psi_{3-k}\left(\rho_{p}\right)}{\sqrt{1-\left(\frac{\gamma_{3-k}-\delta_{3-k}}{2} \rho_{p}+\frac{\gamma_{3-k}+\delta_{3-k}}{2}\right)^{2}}}
$$

Finally we derive the formulas to determine the Stress Intensity Factors S.I.F . Regarding symmetry of the problem due to axis $x=\frac{l}{2}$ we take into consideration only the right hand crack with end points at $x=a_{2}$ and $x=b_{2}$. The stress intensity factors are defined as follows $[1,2,5,12]$

$$
\begin{aligned}
& K_{I I I}\left(a_{2}\right)=\lim _{x \rightarrow a_{2}-0}\left[\sqrt{2 \pi\left(a_{2}-x\right)} \tau_{y z}\right]=\lim _{x \rightarrow a_{2}-0}\left[\sqrt{2 \pi\left(a_{2}-x\right)} \tau(x)\right] \\
& K_{I I I}\left(b_{2}\right)=\lim _{x \rightarrow b_{2}+0}\left[\sqrt{2 \pi\left(x-b_{2}\right)} \tau_{y z}\right]=\lim _{x \rightarrow b_{2}+0}\left[\sqrt{2 \pi\left(x-b_{2}\right)} \tau(x)\right]
\end{aligned}
$$

where $\tau(x)$ are fracture shear stresses that determined according to (2.18) and or (3.2). At first in formula (3.2) by using the first term of relations (2.1) we convert variable $t$ to variable $r$. Then for calculation the S.I.F investigate the below stress function.

$I_{0}(r)=-\frac{1}{\pi} \int_{-1}^{1} \frac{\psi_{1}^{(0)}(\rho) d \rho}{(\rho-r) \sqrt{1-\rho^{2}}} \quad(|r|>1)$.

Here according to [12] we suppose that

$\psi_{1}^{(0)}(\rho)=\psi_{1}^{*}(\rho)+A \rho+B ; \psi_{1}^{*}(\rho)=\psi_{1}^{(0)}(\rho)-A \rho-B$

and select parameters A and B so that $\psi_{1}^{*}( \pm 1)=0$, from which it is concluded that

$$
A=\frac{1}{2}\left[\psi_{1}^{(0)}(1)-\psi_{1}^{(0)}(-1)\right] ; \quad B=\frac{1}{2}\left[\psi_{1}^{(0)}(1)+\psi_{1}^{(0)}(-1)\right]
$$

And consequently

$$
I_{0}(r)=-\frac{1}{\pi} \int_{-1}^{1} \frac{\psi_{1}^{*}(\rho) d \rho}{(\rho-r) \sqrt{1-\rho^{2}}} A+\frac{(A r+B) \operatorname{sign} r}{\sqrt{r^{2}-1}} \quad(|r|>1) .
$$

With the aid of relations (3.6) and (3.7) using in (3.5) and after some simple transforms on it we have

$$
\begin{aligned}
& K_{I I I}\left(a_{2}\right)=\frac{2 G_{1} G_{2} \psi_{1}^{(0)}(1)}{G_{1}+G_{2}} \sqrt{\frac{l(\sin \alpha-\sin \beta)}{2 \cos \beta}} ; \\
& K_{I I I}\left(b_{2}\right)=-\frac{2 G_{1} G_{2} \psi_{1}^{(0)}(-1)}{G_{1}+G_{2}} \sqrt{\frac{l(\sin \alpha-\sin \beta)}{2 \cos \alpha}} ;
\end{aligned}
$$

On the other hand if we consider the dimensionless form of S.I.F in (3.8) obviously it is clear that:

$$
\begin{aligned}
& K_{I I I}^{ \pm}=\frac{G_{1}+G_{2}}{2 G_{1} G_{2} \sqrt{l}}\left\{\begin{array}{l}
K_{I I I}\left(b_{2}\right) \\
K_{I I I}\left(a_{2}\right)
\end{array}\right\} . \\
& K_{I I I}^{+}=-\psi_{1}^{(0)}(-1) \sqrt{\frac{\sin \alpha-\sin \beta}{2 \cos \alpha}} ; K_{I I I}^{-}=\psi_{1}^{(0)}(1) \sqrt{\frac{\sin \alpha-\sin \beta}{2 \cos \beta}} .
\end{aligned}
$$


Quantities $\psi_{1}^{(0)}( \pm 1)$ in above formulas are found through solution of linear system of equations (2.4) taking advantage Lagrange interpolation coefficients.

$$
\begin{aligned}
& \psi_{1}^{(0)}(1)=\frac{1}{M} \sum_{p=1}^{M}(-1)^{p+1} \psi_{1}^{(0)}\left(\rho_{p}\right) \operatorname{ctg}\left(\frac{2 p-1}{4 M} \pi\right) \\
& \psi_{1}^{(0)}(-1)=\frac{1}{M} \sum_{p=1}^{M}(-1)^{M+p} \psi_{1}^{(0)}\left(\rho_{p}\right) \operatorname{tg}\left(\frac{2 p-1}{4 M} \pi\right)
\end{aligned}
$$

In this manner the calculation formulas to determine the problem main characteristics are (3.3), (3.4), (3.9) and (3.10).

\section{Numerical calculations}

To solve the system of linear equations (2.4) we use $M=10,15,20,30, \ldots$ In order that quantities of solutions converge and the different between two latest answers reach to the order $10^{-4}$. For calculation the parameters of function (2.4), $f_{k}(r), L_{k n}(r, \rho), K_{k n}(r, \rho), \delta_{k}, \gamma_{k}$ formulas (2.2), (1.15), (1.16), (3.1) are used.

The parameter $\alpha$ and $\beta$ are supposed equal to $\alpha=\frac{\pi}{3}$ and $\beta=\frac{\pi}{4} ; \frac{\pi}{8} ; \frac{\pi}{16} ; \frac{\pi}{32} ; \frac{\pi}{64}$ in order to determine the influence of distance between two adjacent cracks on their fracture characteristics. The nondimensional shear stress $\tau_{0}(t)$ is calculated according to (3.3), in which the function $f(t)$ is obtained from (3.1), supposing the quantity $Q_{0}=0,01$. Using formulas (3.9) and (3.10) the stress intensity factors in the nondimensional form can be easily calculated, as shown in Fig. 2 and table 1, for above parameters. The variation of S.I.F $K_{I I I}$ based on parameter $\beta$ and $\mu=0.3,0.5,1.0$ is shown in Fig. 3 to recognize the state of S.I.F under change of the lengths cracks.

\section{Conclusions}

The numerical schemes that are used to solve the singular integral equation governing the piecewise homogeneous elastic plate problem subject to uniform remote antiplane shear loading are accurate to determine the tearing shear stresses, cracks dislocation densities and the mode III stress intensity factors. For the case of cracks approaching together it has been shown that the stress intensity factors $K_{I I I}$ grow up based on the two parameters, that are the total distance of their far tips and the closeness of their near tips, for example about 45 percent in the case of $\alpha=\frac{\pi}{3}$ and $\beta=\frac{\pi}{64}$. The technique presented in this paper can be

\begin{tabular}{|c|c|c|c|c|c|}
\hline 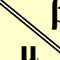 & $\pi / 4$ & $\pi / 8$ & $\pi / 16$ & $\pi / 32$ & $\pi / 64$ \\
\hline 0.3 & 5.36 & 5.54 & 5.73 & 6.17 & 6.35 \\
\hline 0.5 & 4.88 & 5.12 & 5.34 & 5.87 & 6.13 \\
\hline 1.0 & 4.21 & 4.35 & 4.63 & 5.41 & 5.92 \\
\hline
\end{tabular}
used to solve a class of problems associated with the cracking in bimaterials interface.

Table1.Variation of $K_{I I I}$ for $\alpha=\pi / 3$ nbased $o \beta, \mu$ 


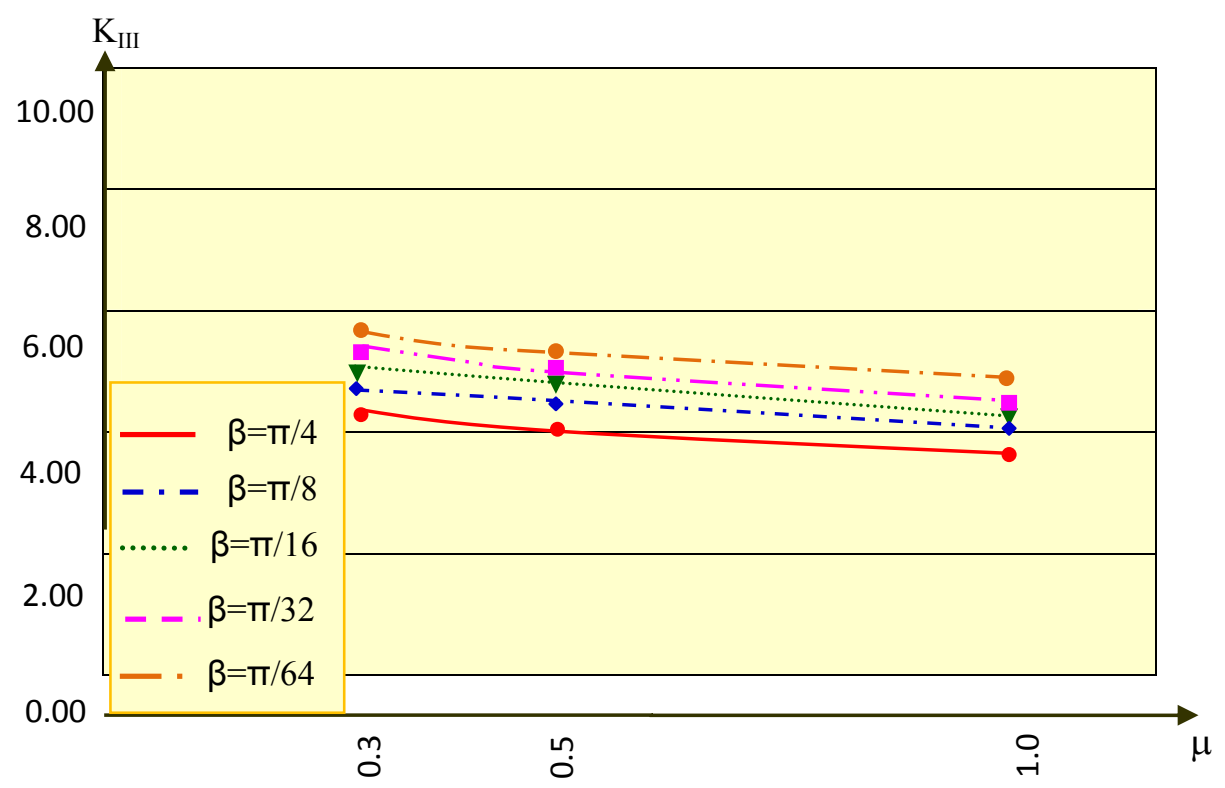

Fig2.Variation of $K_{I I I}$ for $\alpha=\pi / 3$ nbased o $\beta, \mu$

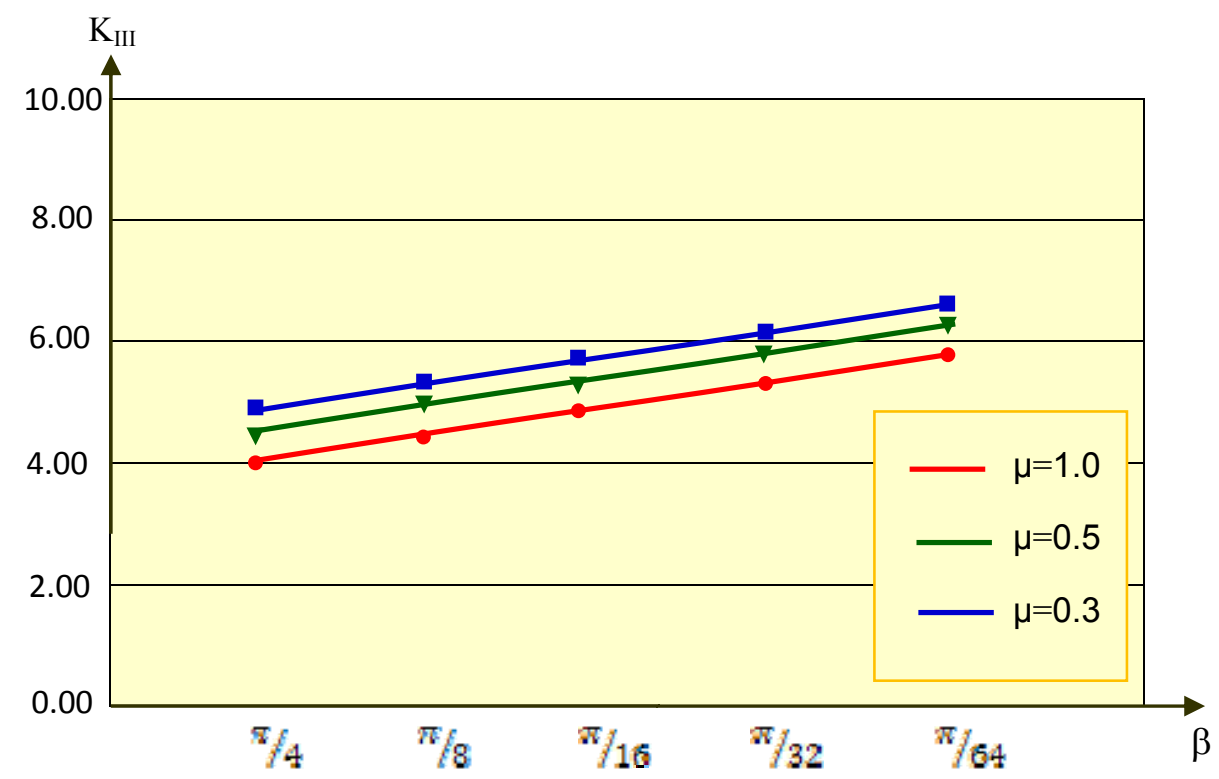

Fig3. Variation of $K_{I I I}$ based $\beta$ for $\alpha=\pi / 3$ and $\mu=1,0.5,0.3$ 


\section{REFERENCES}

1. Fracture an advanced treatise. Vol. II. Mathematical Fundamentals. Academic PressNew York and London, 1968.

2. Cherepanov G.P. Fracture Mechanics. M.: Nauka, 1974.640p.

3. Stress intensity factors. Handbook. Editor-in-Chief Y. Murakami. Vol.1. Pergamon 1987.

4. Savruk M.P. The stress intensity factors in cracked bodies.Fracture Mechanics and strength of Materials. Handbook. Editor V.V. Panasyuk, vol.2, Kiev: Naukova Dumka, 1988.619p.

5. Kachanov L.M. The Principles of Fracture Mechanics. M.: Nauka, 1974. 312c.

6. Panasyuk V.V, Anderekiv P.E, Parton V.Z The principles of Fracture Mechanics. Text book, general editor V.V panasyuk, Vol.1, Kiev: Naukova Dumka, 1988. 488c.

7. Panasyuk V.V., Savruk M.P., Datsyishin A.P. The stress distribution in the neighborhood of cracks of plates and shells. Kiev : Naukova Dumka, 1976. 443p.

8. Theocaris P.S., Iokimidis N.I. Numerical Integration Methods for the Solution of Singular Integral Equations. -Quart. Appl. Math., vol. XXXV, N1, 1977, pp.173-185.

9. Erdogan F., Gupta G.D., Cook T.S. The numerical solutions of singular integral equations. Methods of Analysis and Solution of Crack Problems, pp.368-425, Noordhoff Intern. Publ., Leyden, 1973.

10. Mkhitaryan S.M., Pahlaviani Ali G. The Stress Distribution Field of Piecewise Homogeneous Elastic Rectangular Plate with a System of collinear Cracks Subject to Antiplane Deformations. -2-nd Intern. Scientific and Techn. Conf. "Architecture and Construction- Contemporary Problems" 30Sept. -3Oct. 2010, Yerevan-Jermuk. Conference proceedings, pp.295-303.

11. Sneddon I. Fourier Transforms.- New York-Toronto-London. 1951.

12. Pahlaviani Ali G. Main Characteristics of Fracture Failure of a Piecewise Homogeneous Elastic Plate with Crack Subject to Antiplane Deformations.-2-nd Intern. Scientific and Techn. Conf. "Architecture and Construction- Contemporary Problems" 30Sept. -3Oct. 2010, Yerevan-Jermuk. Conference proceedings, pp.304310.

Name: Ali G.Pahlaviani

Yerevan state university of architecture and construction

Father's name: Eskandar

Address: No.63, Negar Build, 104, West Sarv Blvd, Saadat abad, Tehran, Iran

Tel: +98 21 22141574-6, 22351107-8

Fax: +98 2122060428

E-mail: rahsazehpol@yahoo.com

Поступила в редакцию 24.05.2012 